\title{
10
}

\section{Designing an ODP Trader Implementation using X.500}

\author{
Andrew Waugh ${ }^{\mathrm{a}}$ and Mirion Bearman ${ }^{\mathrm{b}}$ \\ aCSIRO Division of Information Technology, \\ Research Data Network Collaborative Research Centre* \\ bFaculty of Information Science and Engineering, University of Canberra, \\ Collaborative Research Centre for Distributed Systems Technology Centre*
}

This paper describes how an ODP Trader can be implemented using the X.500 Directory Standard. It differs from previous work in this area as we concentrate on the details of the X.500 interface for an ODP Trader, rather than the implementation of the Trader agent itself. We take the ODP specification and describe how the functionality can be supported using X.500. Because of the different data and operations models it is not possible to directly replace an ODP Trader with X.500. Instead, X.500 is used to store the Trader information, including the Service Offers, Links to interworking Traders, and information about the Trader itself. The Trader functionality is implemented using a specialised X.500 user agent, the T-DUA. The TDUA uses the X.500 operations, particularly search, to interrogate and manipulate the Trader database. Use of X.500 in this way simplifies the implementation of the Trader.

Keyword Codes: C.2.4

Keywords: Distributed Systems

\section{INTRODUCTION}

The Reference Model for Open Distributed Processing (RM-ODP) defines a "coordinating framework for the standardisation of ODP by creating an architecture which supports distribution, interworking, interoperability and portability." [5]. The RM-ODP identifies a set of functions that are required to support Open Distributed Processing. One of these is the Trading Function. It is realised by an ODP Trader, which matches objects providing services to objects wishing to use those services. Thus, a trader provides the means for advertising service offers and the means to discover service offers by service requests. Part of the requirements for the progression of the ODP Trading Function standard to Committee Draft status was the inclusion of an Annex which describes how an ODP Trader could be implemented using the X.500 Directory standard. This paper describes the work which led to that Annex.

The underlying data models of both X.500 and the ODP Trader are very similar. Both represent information as type/value pairs. In both, these type/value pairs are collected into objects which can be manipulated as units. This similarity means that much of the complexity of an ODP Trader implementation can be avoided if X.500 is used to store the Trader information.

Our design principle was to attempt to make maximum use of the features of X.500 while implementing exactly the functionality required by an ODP Trader. However, we quickly recognised that the functionality required by the Trader was more sophisticated in many areas than could be directly supported by X.500. For example, Trader service properties may be 'indirect', that is, the Trader does not store the value of the service property, but retrieves the

The work reported in this paper has been funded in part by the Cooperative Research Centres Program through the Department of the Prime Minister and Cabinet of the Commonwealth Government of Australia 
value from the Exporter when required. Such differences in functionality led to an implementation design that uses X.500 as a database to store the Trader Information Object. A special Directory User Agent, the Trader DUA (T-DUA), implements the functionality required by an ODP Trader on top of X.500. This permits maximum use of the features of X.500 while still implementing the functionality required by an ODP Trader.

The X.500 Trader Information Object stores the information in one of four types of entry: the Trader Entry, which stores information about the characteristics of the Trader itself,

$\dagger \quad$ the Trader Policy Entry, which stores information about the policies which govern the behaviour of the Trader,

$\dagger \quad$ the Trader Link Entries, which store information about other Traders with which this Trader interworks,

$\dagger \quad$ the Service Offer Entries, which store information about the Service Offers known to this Trader.

The X.500 protocol is used to search and manipulate this information. The use of X.500 in this fashion means that the T-DUA does not have to implement an object based database, nor to implement functions to perform complicated manipulations on objects in this database. This simplifies the implementation of an ODP Trader.

The functionality which could not be implemented directly using X.500 includes:

$\dagger \quad$ The Trader Operations. The T-DUA is responsible for mapping each Trader Operation into one or more X.500 operations.

$\dagger \quad$ Indirect Properties. These are retrieved by the T-DUA which is consequently responsible for the final evaluation of candidate Service Offers retrieved from the X.500 Trader Information Object.

$\dagger \quad$ The Selection Preference. As X.500 provides no support for ranking search results, the evaluation of the selection preference is the responsibility of the T-DUA.

We plan to implement the Trader design presented in this paper. Apart from validating our design, this will assist in defining and clarifying concepts in an ODP Trader specification. These include, for example, the functionality required of: access control, the Type Manager, and interworking (federation) of Traders.

\section{ODP TRADER}

The concepts and terms used to describe the Trader and its interactions in this paper are taken from the ODP Trading Function Standard [1]. The following description introduces this Trader model. Only those concepts needed in this paper are described.

An object which advertises a service for use by other objects exports a description of that service to a Trader. This effectively forms an advertisement for that service. The Trader stores these description in its Trader Information Object. Importing involves an object needing a service sending a description of the desired service to the Trader. The Trader searches its Information Object for matching Service Offers. These are returned to the importer, which selects one and connects directly with the server. The Importer may request the Trader to sort the returned service offers, or even select the 'best'.

A Service Offer describes the type of service on offer and the Properties associated with that service. In addition, a service offer can include Service Offer Properties that are associated with the offer itself. For example, a service offer of a printing service can have a Service Property describing the quality of the output and an Offer Property describing the expiration date of the offer. The properties are described by name/value pairs where several values may associate with a name. The properties which can be included in a particular Service type are controlled by the Type Manager, which is logically separate from the Trader.

Some properties included in a particular Service Type may be defined in the Type Manager to be indirect. The Exporter does not include values for these properties when a Service Offer is exported. Instead, it specifies an interface at which the values can be dynamically obtained. This interface is known as the Service Offer Evaluation Interface (SOE Interface). When the 
Trader needs to evaluate whether or not a particular Service Offer satisfies a particular Import request, it contacts the SOE Interface and retrieves the values of the indirect properties.

Traders may be linked together so that an Import request to one Trader may result in the return of a Service Offer exported to a different Trader. This is referred to as interworking. To enable interworking, a Trader also stores a database of Trader Links in addition to the database of Service Offers. Links contain properties which describe the linked Traders. These properties are evaluated when a Trader decides to follow a link to another Trader when attempting to fulfil an Import request. Figure 1 shows the interactions between the Importer, Traders, and Exporters.

The Importer can specify the following in an import request:

$\dagger \quad$ Service Description - specifies the type of Service required.

$\dagger \quad$ Service Property Names - specifies a list of Service Properties to be returned with the selected Service Offers. The default is no Service Properties to be returned.

$\dagger \quad$ Service Offer Property Names - specifies a list of Service Offer Properties to be returned

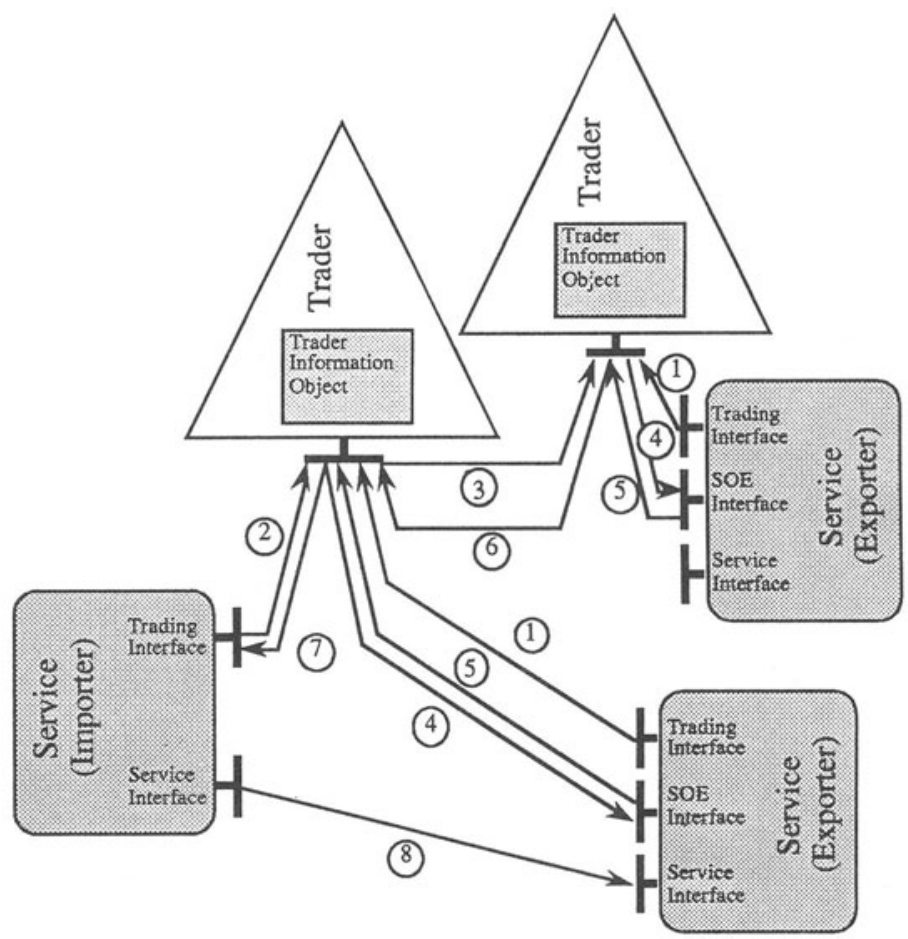

Figure 1. The interactions between an Importer, Exporter and Traders. 1: Exporter exports Service Offer to Trader. 2: Importer requests Service Offer. 3: Trader forwards Import request to Interworking Trader. 4: Trader retrieves values of indirect properties. 5: Exporter responds with indirect properties. 6: Interworking Trader responds with Service Offers. 7: Trader responds with ordered list of Service Offers. 8: Importer requests service from Server. 
with the selected Service Offers. The default is no Service Offer Properties to be returned.

$\dagger \quad$ Import Policy Constraint - describes the expectations of the scope of the services imported. Little detail is specified for this constraint in the Trading Function Standard. We found it useful to separate Import Policy Constraints into three categories, which we have named:

- Importer Trader Constraints. These are constraints on the Trader using Trader properties, for example the ownership of the Trader. If the Trader cannot satisfy these constraints it does not undertake the Import request

- Importer Link Constraints. These are constraints on any Trader which is passed the Import request by the original Trader when interworking. Again, an example is the ownership of the linked Trader. These constraints are expressed as link properties and are applied by the first Trader, not by the interworking Trader.

- Importer Import Constraints. These are constraints on the search of the local Service Offers, for example an upper bound on the number of offers to be returned.

$\dagger \quad$ Matching Constraint Criteria - specifies the filter to be applied to the Service Offers of the desired type to determine whether the Offer is of interest. This is a boolean expression on the Service and Service Offer properties.

$\dagger \quad$ Selection Preference - specifies an ordering function to be applied to the matched Service Offers. This function specifies which matched Service Offers are to be preferred. This is an expression on the Service and Service Offer properties.

\section{X.500}

$\mathrm{X} .500[2,6]$ is a standard that allows the construction of very large, distributed, directories. An X.500 directory is implemented using two entities: DUAs (Directory User Agents) and DSAs (Directory System Agents). The DSAs are the servers and store the directory information. The DUAs are the user agents.

X.500 is a directory and stores information about objects: in a Trader, objects include Service Offers, Trader Links, and the Trader itself. These objects are represented as entries. Each entry contains the information about one object. Entries are equivalent to records in a conventional database. The information in an entry is represented by attributes. Each attribute contains a fact about the object. Typical facts are the object's name, it's location, and description. Attributes are similar to fields in a conventional database; however they have some differences. An attribute may have more than one value. The telephoneNumber attribute, for example, can store several telephone numbers. The attributes that may be contained or must be contained in an entry of a particular type are controlled by the X.500 schema. Changing the schema does not require shutting down the entire directory, nor need the entire X.500 schema be changed at once. X.500 allows new attributes and types of entries to be defined at any time.

Entries are arranged hierarchically and form nodes in a Directory Information Tree (DIT). Typically, this tree can reflect the structure of the directory information. Within an organisation, for example, the tree can represent the organisational hierarchy with the following levels: country, organisation, organisational units, staff. Underneath the root of the tree, for example, there can be entries for each country. Then, under a country entry there can be entries for each organisation in the country. This hierarchical organisation allows the directory information to be distributed amongst the many DSAs (servers), and yet still allows quick identification of the server which holds a particular entry.

X.500 provides operations that allow (among other things) a user to read the contents of an entry, to search a portion of the tree for entries which satisfy certain criteria, to create entries, and to modify the contents of an entry.

\section{RATIONALE}

Using X.500 to support an ODP Trader has some significant attractions. These include: 
The re-use of existing infrastructure. X.500 implementations exist and are being deployed in organisations. A significant amount of investment has already been made in X.500 technology by both software developers and organisations using X.500. Using X.500 as a base to support an ODP Trader allows us to exploit this investment, potentially significantly reducing the cost of deploying an ODP Trader system.

$\dagger \quad$ The underlying information model supported by X.500 is similar to that required by an ODP Trader. The concept of X.500 attributes, for example, is very similar to the Trader concept of properties. In particular, an X.500 attribute may contain multiple values, which is different from most conventional databases.

$\dagger \quad$ X.500 was designed to allow administrators considerable flexibility in changing the schema without either needing to bring down the directory, or requiring that the schema be changed throughout the distributed directory at one time. This would allow similar flexibility in the administration of an ODP Trader Service Type definitions.

$\dagger \quad$ An ODP Trader could use the general X.500 infrastructure to look up network addresses of linked Traders and Clients. It could also use the security features of X.500 to authenticate users and control access to Trader information.

If an existing X.500 system is used as the base for the implementation of an ODP Trader, it is unnecessary to re-implement these functions in the Trader. This significantly simplifies the work of implementing the ODP Trader.

The goal of this project was to design an ODP Trader implementation using X.500. Thus, the functionality to be supported was fixed by the Trader group and we could not change it. Similarly, the functionality supplied by X.500 was also fixed. The design has to map between what is supplied by X.500 and what is required by an ODP Trader specification. In our design, we aimed to maximise the use of X.500 functionality.

\section{USING X.500 AS THE TRADER}

Our first design option was to use X.500 as the Trader. Unfortunately, this option was not feasible as the model used by X.500 and the model used by the ODP Trader differ significantly in a number of ways. These include:

$\dagger$ The functional model. The fundamental unit in X.500 is the entry. The X.500 operations consequently operate on entries, for example, reading information from the entry, adding new entries, or searching for entries which match a specified filter. The fundamental units in an ODP Trader model are Service Offers, Trader Links, Trader Policies and Trader Properties. The Trader operations consequently manipulate these units. There are several distinct operations which add information to the Trader Information Object. Examples of these operations are: Export, Add Link, New Trader Property and New Trader Policy. X.500, on the other hand, has just one such operation: AddEntry. It is consequently necessary to map between the Trader protocol and the X.500 protocol.

$\dagger \quad$ The method for supporting distributed operations. In X.500 the distribution of information amongst the servers is fixed by the standard and is based on the hierarchical structure of the tree. The user has no control on which server a particular entry is placed: the user specifies the entry's DN and the entry is placed on the server which stores that particular portion of the tree. All.DSAs in an X.500 directory co-operate to implement this single hierarchical distribution mechanism. Distribution in an ODP Trader is much less fixed. The user exports a Service Offer to a specific Trader. The administrator of that Trader may arrange for it to interwork with other Traders, in which case the exported service is available to a greater range of users. The standard, however, does not specify any structure for these interworking Traders.

$\dagger \quad$ The source of information. All information in an X.500 directory is assumed to reside on $X .500$ servers. The server could 'hold' this information on another database and retrieve it when required, but this is transparent to the X.500 protocol. Properties in a service offer held by an ODP Trader, however, may be specified to be 'indirect' and the values of these properties are not held by the Trader. When it is necessary to evaluate the service 


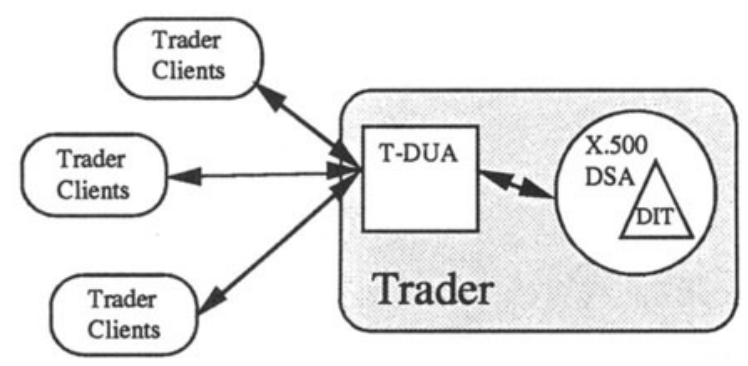

Figure 2: The Trader with its components and clients

offer (during an Import, for example), the Service Offer Evaluation Interface is called to obtain the current values of those properties.

$\dagger \quad$ Ordering of Results. An importer may specify how the returned set of Service Offers is to be sorted. X.500 does not support the ordering of a set of results.

\section{USING X.500 AS THE TRADER DATABASE}

\subsection{Trader structure}

The approach adopted was to implement an ODP Trader as two components, a combination of an X.500 DUA and an X.500 DSA. The DSA stores the Trader Information Object (i.e. the information that the Trader knows). This information is accessed by the T-DUA (Trader DUA). The T-DUA implements the aspects of an ODP Trader specification which cannot be directly supported using X.500. Requests from trader clients (importers, exporters, and administrators) are mapped by the T-DUA into operations on the X.500 database. Figure 2 shows the components of an ODP Trader and its interactions with the clients.

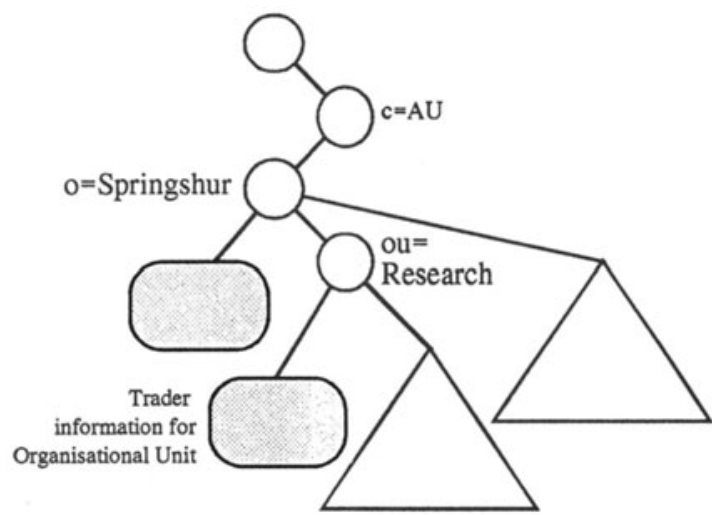

Figure 3: Placement of the Trader Information Object within the X.500 global directory. 


\subsection{Proposed X.500 directory information tree}

The information stored by the X.500 directory includes:

$\dagger \quad$ The Trader properties (i.e. information about the Trader itself),

$\dagger \quad$ The Trader policies (i.e. rules to determine and guide Trader behaviour)

$\dagger \quad$ The set of Service Offers (i.e. information used by the Trader when acting as a server to undertake import or export operations)

$\dagger \quad$ The set of Trader Links (i.e. the information used by the Trader when acting as a client accessing other Traders when undertaking an import operation)

$\dagger \quad$ Type Manager Information (i.e. the information used by the Trader to control the form of Service Offer exports)

The Type Manager, and its information, are conceptually separate from the Trader and will be discussed in a later section of this paper.

The remaining four categories of information (trader properties, trader policies, service offers, and trader links) collectively form the Trader Information Object. This information is represented as a subtree of the X.500 directory tree. The subtree can be attached anywhere in the X.500 tree, but we expect that the Trader subtree would be most commonly attached beneath entries representing Organisations and Organisational Units (representing, respectively, the information known to organisational and organisational unit traders). This is shown in Figure 3.

Note that no attempt is made to link these packages of information together using the inherent X.500 distribution mechanism. Interworking between Traders is solely performed by the T-DUA using the Trader Link information.

Each Trader Information Object is composed of four types of X.500 entries. For simplicity, these entries are arranged in a flat tree as shown in Figure 4. The four types of entries are:

$\dagger \quad$ The Trader Entry contains information about the Trader itself. The information consists of:

- Trader Properties, such as the owner of the Trader

- Configuration Information, such as the name of the Trader and its network address

- Access control information

- $\quad$ Authentication information for the Trader Administrator

- Limit information, such as the amount of resources to be consumed in an Import search.

$\dagger \quad$ The Trader Policy Entry contains the details about the Trader policies and is located immediately underneath the Trader Entry. The entry contains the trader policies,

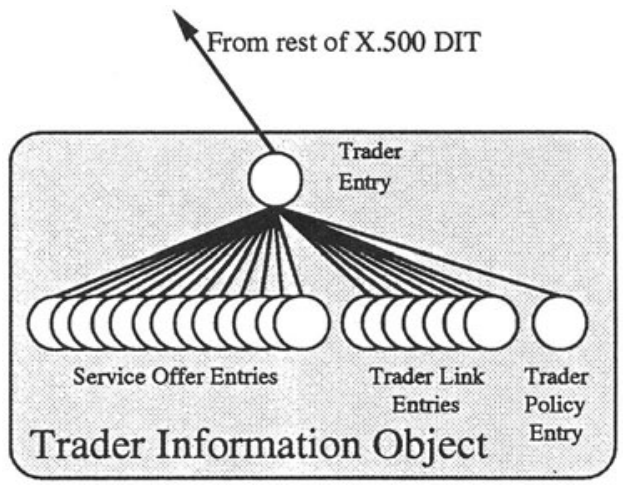

Figure 4: How the Trader Information Object is represented using X.500 entries. 
expressed as a collection of X.500 attributes. Logically, this information is actually part of the information about the Trader and so should be stored in the Trader entry. Storing the Trader policy information in a separate entry, however, simplifies implementation of the separate Trader operations which manipulate Trader Policies and Trader Properties. If both the policies and properties were stored in one entry it would be difficult to ensure that a Trader Policy operation could only manipulate policies and not properties (and vice versa).

$\dagger \quad$ A Service Offer Entry contains the information about one Service Offer. Service Offer entries are also located immediately beneath the Trader Entry. The basic attributes which are contained in each Service Offer entry are:

- Name: The name of the Service Offer, assigned by the Trader

- Exporter: The X.500 name of the Exporter of the service

- ServiceInterface: The X.500 name of the interface of the service being offered

- ServiceDescription: An X.500 auxiliary object class identifier

- SOEInterfaceId: The X.500 name of the interface from which the current value of the indirect properties are to be retrieved.

In addition to these basic attributes, a Service Offer entry contains attributes which represent the Service Properties. These properties depend on the type of service the Service Offer describes. The Service description is represented by a Service Type Identifier in this implementation. This is represented by an X.500 auxiliary object class that controls (via the X.500 schema) which attributes (i.e. properties) must be present and which attributes may be present in the Service Offer.

The Service Offer may contain Service Offer Properties. These are also represented as X.500 attributes in the Service Offer entry. Depending on the Trader Importer Policy these Service Offer Properties may contain ValidFrom and ValidTo properties which define the period over which the Service Offer is valid.

The Service Offer entry only contains direct properties. The handling of indirect properties is implemented by the T-DUA. This will be described in the section on Import and Export.

Finally, the Service Offer entry may contain other attributes which contain access control information, human readable information about the service offer (e.g. a description), and rules that describe an exporter's expectations of a service offer (i.e. exporter policy).

We considered structuring the Service Offer Entries into a tree based on a service hierarchy. However, this did not produce any functionality required by the Trader, nor did it provide any implementation advantages.

$\dagger \quad$ A Trader Link Entry contains the information about one Trader Link. A Trader Link entry contains:

- Name: The name of the Trader Link. This is assigned by the Trader

- TraderInterface: The X.500 name of the linked Trader.

It also contains a set of Trader Link properties, which are expressed as attributes. The Trader Link entry may contain other attributes which contain access control information, human readable information about the trading service offer of the linked Trader, attributes from the linked Trader Entry, and the period over which this link is active. How these properties will be used to find Service Offers on remote Traders is currently not defined in the RM-ODP standard. This is one area we wish to investigate in the future.

\section{TYPE MANAGER}

It was decided not to design a sophisticated Type Manager, primarily because it was not clear from the ODP Trader document exactly what functions a Trader would require of a Type Manager. Also, the Type Manager is conceptually separate from the Trader and hence outside the strict scope of our work. It was necessary, however, to design a minimal Type Manager which would be able to perform the following functions: 
$\dagger \quad$ Control the Service Properties and Service Offer Properties present in a Service Offer. This involves ensuring both that mandatory properties are present and that the exporter has not included any extraneous properties. This part of the Type Manager is handled by the X.500 schema which can control the mandatory and optional attributes (properties) present in an entry (Service Offer) of a particular object class (Service Description).

$\dagger \quad$ Store the set of indirect properties to be included in a Service Offer of a particular type. This is handled by defining a new type of X.500 entry which represents Type Manager information. One such entry exists for each different type of Service defined. Each entry includes two attributes. One contains the names of any mandatory indirect properties (i.e. those that the Service Offer must supply) and the other contains the names of any optional indirect properties (i.e. those that an Service Offer may supply):

These entries could form the basis of a more sophisticated Type Manager. (These entries are not shown in figure 4 as they are not part of the Trader Information Object.)

We intend to extend this minimal Type Manager as we determine what functions are required by an ODP Trader.

\section{TRADER OPERATIONS}

To give the reader an idea of the interactions between the T-DUA and the DSA, we describe the Import and Export Operations. The description is a fairly high level overview, intended to highlight the issues involved in implementing the ODP Trader.

\subsection{Export}

The Export Operation adds a Service Offer to the Trader Information Base. Specifically, the Export Operation is mapped onto an X.500 Add Entry operation to add a new Service Offer entry to the X.500 database.

The mapping is straightforward since the Service Description is an X.500 object class and the Service Properties and Service Offer Properties are X.500 attributes.

It is necessary to check that the exported Service Offer conforms to the template in the Type Manager. This check is performed in two parts. The first part is undertaken directly by the TDUA, which reads the template entry for that Service Type from the Type Manager (using X.500). A successful read operation confirms that the Trader does handle this type of Service Offer and indicates whether this Service Type has indirect properties. If indirect properties exist then the T-DUA checks that the Service Offer includes the mandatory indirect properties and is not proposing to include any extraneous optional indirect properties (note that the Trader does not store which optional indirect properties the Exporter is providing at the SOE Interface). All the indirect properties are then stripped from the Service Offer and the result is then stored in the X.500 database. If the Service Type includes indirect properties, the T-DUA checks that the Service Offer includes an SOE Interface.

The second part of the check is an X.500 schema check. This checks that, given the Service Description, the Service Offer contains all the mandatory properties and does not contain any extraneous properties. This check occurs when T-DUA attempts to add the Service Offer entry to the X.500 database.

A Service Offer is added to the database only if it passes both checks.

\subsection{Import}

Import is a complicated Trader operation because of:

$\dagger$ The necessity to check the Importer Policy

$\dagger \quad$ The potential for searching interworking Traders

$\dagger \quad$ The presence of indirect attributes in the Service Offers.

The first step in processing an Import request is for the Trader to decide if the Importer Trader Constraints allow it to answer the request at all. This is achieved by the T-DUA performing an X.500 Search Operation on the Trader entry using the Importer Trader 
Constraint as the filter. If the search operation is successful, then the Trader satisfies the constraint.

The second step is to decide if any of the Interworking Traders should be searched. This is achieved by the T-DUA performing a second X.500 search. Here, the target entries are the Trader link entries and the filter is the Importer Link Constraints. A set of Trader Links (possibly empty), which satisfy the Importer's constraint, are returned. The Import operation should be forwarded to these linked Traders, which may be done in parallel to the local search, before the local search, or after it.

The third step is to perform a search of the local database of Service Offers to see if any of them will satisfy the Import request. The search can be complicated by the presence of indirect properties. As these are not stored with the Service Offers, evaluating the Matching Criteria must be a three phase process:

1) The T-DUA retrieves from the Type Manager (using an X.500 read) the list of indirect properties in a Service Offer for this particular Service Type.

2) The Matching Criteria are rewritten to produce a new filter that does not include any tests on indirect properties. This filter finds all the Service Offers which might satisfy the Import request. An X.500 search is then performed for Service Offers that satisfy the requested Service Description and the rewritten filter. A set of potential matches is returned.

3) The Matching Criteria are then rewritten to produce a new filter which only includes the indirect properties. For each Service Offer returned in the previous phase, the specified SOE Interface is contacted and the values for the necessary indirect properties are obtained. The indirect property values are applied to the rewritten filter and that Service Offer is either accepted or eliminated from the set of matches.

The fourth step is to combine the set of Service Offers found locally with the set of Service Offers found though searching the linked Traders, and to rank these Offers using the Selection Preference. The fifth and final step is to select the 'best' matches using the Selection Preference. This may involve evaluating indirect properties. The selected matches are then returned to the Importer.

\section{A SIMPLE EXAMPLE}

To define a new type of service, the Trader administrator has to construct the X.500 schema definitions for the new service properties associated with the new service type. For example, the X.500 schema definition for the properties of a printer service type might be:

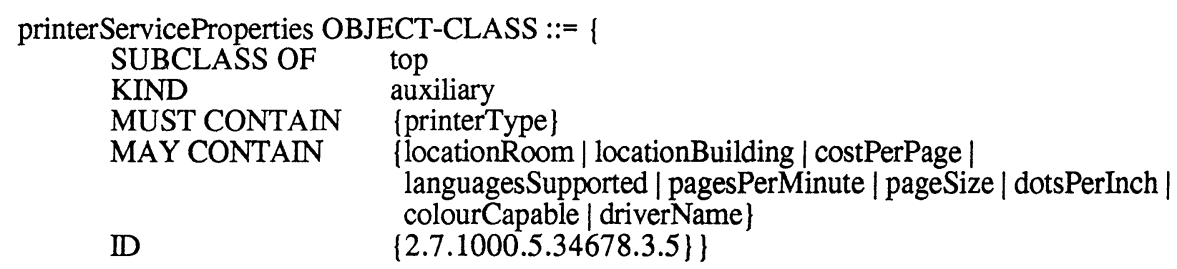

This definition states that an exported offer of a printer service must contain one property ('PrinterType'), and may contain ten other properties, including 'costPerPage'. Properties are defined as X.500 attributes, the 'costPerPage' property, for example, might be defined as:

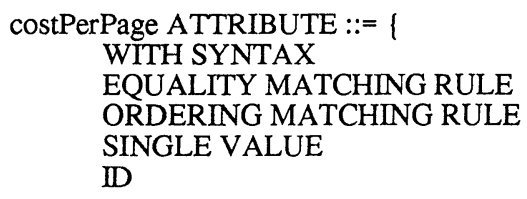

$\{2.7 .1000 .5 \cdot 34678.4 .1\}\}$ 
which states that a 'costPerPage' property contains a single integer. With most X.500 DSA implementations, adding such simple definitions only involves editing configuration files and restarting the DSA. The attribute definitions also need to be added to the T-DUA configuration files and the T-DUA restarted.

When an entry is created in the X.500 database representing an actual Service Offer for a printer service, the entry will contain a number of additional attributes. These represent the Service Type and Service Offer Properties and include, for example, the Service Interface Identifier, the identity of the Exporter, and the period of validity of the Service Offer.

Finally the Type Manager entry needs to be added to the X.500 Database. This entry only contains the name and identifier of this new service.

Importers can search for these offers by specifying a Service Description of the OID '2.7.1000.5.34678.3.5' (which is usually aliased to a more user-friendly string such as 'printerServiceOffer' in the T-DUA) and a Matching Criteria, such as

printerType $=$ "Laserwriter" and dotsPerInch $>600$ and languagesSupported $=$ "Postscript"

\section{INDIRECT PROPERTY EXAMPLE}

Assume that the importers wished to check whether a print service was actually available (i.e. not out of paper, out of toner, or jammed). This could be represented by an indirect property 'printerAvailable'. It is not necessary to modify the 'printerServiceProperties' definition given in the previous example as the information to be stored in the X.500 database for each Printer Service offer will not be changed. However, the Service Type entry for the Printer Service must be altered to include the new indirect attribute. The contents of this entry might be:

$$
\begin{array}{ll}
\text { IcommonName } & =\text { printerService } \\
\text { serviceType } & =\{2.7 .1000 .5 .34678 .3 .5\} \\
\text { indirectProperties } & =\text { printerAvailable }\}
\end{array}
$$

To import a print service, the user might specify the following Matching Criteria:

printerType = "Laserwriter" and languagesSupported = "Postscript" and printerAvailable

The T-DUA first reads the printerServiceOffer Service Type entry and discovers that 'printerAvailable' is an indirect property. It rewrites the Matching Criteria to remove that property to give

\section{printerType $=$ "Laserwriter" and languagesSupported = "Postscript"}

Evaluating this X.500 query will return information about all the Print Services matching the filter known to the Trader. The T-DUA must then contact the SOE Interface of each of these offers to determine whether the 'printerAvailable' property is true, and hence whether the Service Offer is actually selected by the Matching Criteria.

\section{RELATIONSHIP TO OTHER WORK}

A number of papers have proposed, or developed, implementations of Traders using X.500. These include Popien \& Meyer [3], and Pratten et al [4]. Unfortunately, other papers are confidential and their contents cannot be publicly disclosed. The overall design of the Traders described in $[3,4]$ is similar to that described in this paper. X.500 is used for the storage of information, while the Trader functionality is implemented using the equivalent of the T-DUA. Popien \& Meyer specifically identify the difference in distributed operations between X.500 and an ODP Trader as a reason for this design.

Both Popien \& Meyer and Pratten describe the implementation of an ODP Trader using X.500. However, there is little overlap between this paper and the earlier papers as both earlier papers concentrate on the internal design of the equivalent of the T-DUA and give little detail on the X.500 interface. Furthermore the operations supported by the Traders are different from 
those in this paper, although this is probably explained by the development of the ODP Trader model subsequent to the writing of the earlier papers.

\section{CONCLUSIONS}

It appears to be possible to implement an ODP Trader using X.500 as a base. X.500, however, is not powerful enough to directly implement the functionality required by an ODP Trader, and so cannot replace an ODP Trader. Instead, it is necessary to provide a special X.500 DUA, which we call the T-DUA, to implement these additional functions. Among other things, the T-DUA implements:

$\dagger$ Support for the different operational models used by the Trader and X.500, including mapping between the X.500 and ODP protocols.

$\dagger \quad$ Support for the distributed Trader operations (interworking).

$\dagger \quad$ Support for indirect attributes.

$\dagger \quad$ Support for ordering the Service Offers returned from an Import operation.

The underlying data models of both X.500 and an ODP Trader are very similar. Information in the Trader is represented as Properties which have a type and one or more values. This directly maps into the equivalent X.500 attributes. The collections of properties and policies in the Trader (Service Offers, Trader Links) map easily into X.500 objects. The X.500 operations for accessing, searching, and modifying information provide similar functionality to those functions required by the Trader.

The use of X.500 to support an ODP Trader consequently should mean a significant simplification in the implementation of the Trader. This is particularly so as X.500 products are now available off the shelf. In addition, using X.500 provides access to the features of X.500 which are currently poorly defined in the Trader. This includes authentication of users, access control of information, methods for modifying and maintaining the underlying data schema, and methods for mapping names of objects in the network to addresses.

We have presented a description of how the Trader Information Object can be represented in the X.500 Directory Information Tree, and have sketched how the Import and Export operations can be implemented.

The exercise of designing an implementation of the ODP Trader has also highlighted areas of the Trader document which need more definition, for example, the specification of the Importer Policy. Ultimately, however, it is an open question as to how detailed the Trader specification should become. Where does defining a general model end, and defining a specific implementation start?

The work described in this paper is continuing. We plan to implement the T-DUA. Apart from validating the design, a prototype will assist in validating the specification of the ODP Trader. We also hope to extend areas of the design, particularly those concerning the Type Manager.

\section{REFERENCES}

[1] ODP Trading Function, ITU/ISO Committee Draft Standard ISO 13235/ITU.TS Rec.9tr (1994) (To appear)

[2] Information Technology - Open Systems Interconnection - The Directory, ITU/ISO Standard X.500/ ISO 9594 (1994) (To appear)

[3] Federating ODP Traders: An X.500 Approach, C. Popien, B. Meyer, Proc. of the ICC'93, Geneva, Switzerland, May 1993.

[4] Design and Implementation of a Trader-Based Resource Management System, A. Warren Pratten, James W. Hong, J. Michael Bennett, Michael A. Bauer, Hanan Lutfiyya, (Private Communication).

[5] Reference Model of Open Distributed Processing: a Tutorial, Kerry Raymond, Open Distributed Processing II, ed J. de Meer, B. Mahr, and S. Storp, North-Holland (1994)

[6] X.500, the directory standard and its application, Douglas Steedman, Technology Appraisals Ltd (1993) 